\title{
A method for making a fair evaluation of driving styles in different scenarios with recommendations for their improvement
}

\author{
Víctor Corcoba Magaña, Xabiel G. Pañeda, Alejandro García Tuero, Laura Pozueco, Roberto García, \\ David Melendi, Abel Rionda
}

\begin{abstract}
Evaluating driving from the point of view of efficiency is a complex task. The environment is continuously changing and there is no direct relationship between all the factors that affect fuel consumption. In this paper, we propose a method to evaluate driving that can fit any scenario. It is based on Data Envelopment Analysis (DEA). This technique allows us to measure the efficiency of the drivers and to ascertain which behaviors they should improve. There are many solutions to evaluate driving in the literature. However, these solutions have difficulties adapting to changes in the driving environment and the result of the evaluation could be inaccurate. A driver could be classified as less efficient when in fact the driving could be affected by factors beyond his or her control. In addition, they do not provide the sources of inefficiency. With our method, we can analyze and quantify the cause of inefficiency. This proposal has been used to evaluate drivers of a bus fleet in Spain. The results show that drivers who were rated as efficient achieve lower fuel consumption. They can save up to $9.21 \%$ in comparison with the least efficient drivers. It is also observed that anticipation is a skill that saves a large amount of fuel and that it is an aspect that most bus drivers have a lot of room for improvement.
\end{abstract}

Index Terms - Eco-Driving Training, Data Envelopment Analysis, Public Transport, Intelligent Transportation System

\section{INTRODUCTION}

A ir pollution is currently a very serious problem that has consequences for health, the economy and the environment. According to [1], heart disease and strokes are responsible for $80 \%$ of the cases of premature death, and they are attributable to air pollution. It increases the likelihood of suffering other respiratory and cardiovascular diseases, cancer, fertility problems, obesity, and diabetes [2] [3]. The main causes of pollution are power generation and road transport [4].

Different measures have been promoted in the transportation area in order to reduce the emission of gaseous and particulate pollutants such as improving the technologies related to

\footnotetext{
${ }^{\mathrm{T}}$ his paragraph of the first footnote will contain the date on which you submitted your paper for review. It will also contain support information, including sponsor and financial support acknowledgment. This work has been supported by the General Direction of Traffic (DGT) of Spain through the project SPIP2015-01686, the Spanish National Research Program within the project TIN2013-41719-R and the Science, Technology and Innovation Plan of the Principality of Asturias, within the project GRUPIN-14-065.
}

aerodynamics or engines (fleet renewal), stimulating public transport, choosing the best route (eco-routing) or changing the driver's behavior (eco-driving).

Eco-driving stands out among the above solutions because it can be applied to any type of vehicle. However, it is very difficult to change people's driving habits. Driving is a complex task because the driver has to carry out several actions at the same time.

Many transportation companies are training their workers in order to improve their driving style [5] [6] [7]. The drivers attend eco-driving courses where they learn the main driving rules to minimize energy consumption. In addition, efficient driving experts supervise the drivers on a real route and advise them about which aspects of driving need to be improved.

In these cases, there are three main problems: the cost of ecodriving classes, the number of eco-driving students, and the subjectivity. The first drawback causes the number of classes to be reduced. The trainer has to detect driver errors in a short time. In addition, the transport companies often have many drivers. The trainer needs to issue customized instructions for each participant. Furthermore, people can be susceptible and do not easily accept criticism, especially if is not well justified.

Apart from the above-mentioned, it is also very important to encourage the drivers to improve their driving behavior. Many authors highlight the need to continuously motivate the drivers because many of them consider eco-driving styles "boring" or they forget or do not feel motivated to apply their knowledge[7].

In order to motivate the drivers and help driving experts, we need a solution to assess the driving. The evaluation method should be accurate and able to provide enough information to identify in which areas the driver needs to improve.

Our research group has been working in conjunction with ADN Context-aware Mobile Solutions S.L to develop a tool to help driving experts [8]. The tool provides the experts with information about the driving habits of bus drivers to enhance

V. Corcoba Magaña, X. G. Pañeda, A. G. Tuero, L. Pozueco, R. García, and D. Melendi, are with the University of Oviedo, Oviedo 33003, Spain (e-mail: corcobavictor@uniovi.es; xabiel@uniovi.es;garciatalejandro@uniovi.es; pozuecolaura@uniovi.es; garciaroberto@uniovi.es; melendi@uniovi.es).

A. Rionda is with ADN Mobile Solutions, Gijón 33204, Spain (e-mail: abel.rionda@adnmobilesolutions.com). 
the eco-driving classes. The trainer can focus on the deficiencies observed in the driving style. This system uses several metrics to show the driver's behavior from the point of view of energy efficiency. It also assigns a score to the drivers based on fuzzy logic and eco-driving rules. This score is used to create a ranking in which the drivers try to obtain a high position. The proposal has been used in bus fleets in Spain and Morocco.

We have observed that the metrics and the fuzzy logic system proposed in [8] do not always produce good results. Evaluating driving is not a trivial matter. There are a large number of variables that can influence results and some do not depend on the driver. Sometimes the evaluation is inaccurate because the algorithm is not able to model the driving scenario. In [8] we have seen that there are drivers who obtain high scores (using the fuzzy logic system) in general taking into account all the bus lines where they drive. But, there are bus lines where the drivers get abnormally low scores. This low score is due to the fact that the fuzzy logic model is not adjusting properly to this bus line. Setting the thresholds for the membership functions of the fuzzy model is not an easy task.

In this work, we propose a method to evaluate bus drivers based on Data Envelopment Analysis (DEA) [9] [10]. This solution does not require to fix the limits considered as permissible to establish that a driver is efficient (e.g.: fuzzy logic) or to label samples to train the algorithm (e.g.: support vector machine). The use of DEA allows us to easily adjust the evaluation criteria. The result is a more accurate and fair assessment of driving behavior. This is important for greater acceptance of the results and to increase motivation. A fair assessment system would allow us to develop a reward program. In addition, the solution identifies the skills that the driver should improve to reduce fuel consumption, enabling the driving expert to focus on them.

The rest of the paper is organized as follows. Section 2 describes the state of the art. Section 3 introduces the proposal. In Section 4, the solution is used to evaluate a bus fleet in Spain. Finally, our conclusions and future work are presented in Section 5.

\section{RELATED WORK}

Eco-driving techniques for reducing fuel consumption have been widely tried in buses. In [11], the researchers conducted a real test with 54 drivers. They introduced two initiatives (an educational program and driving assistant) in the bus line in order to make a comparison. Drivers saved on average $6.8 \%$ of fuel. The results were similar in the two cases. In addition, the authors observed a large decrease in hard deceleration and speeding. However, they also concluded that there are external factors that limit drivers' ability to follow the eco-driving recommendations such as the weather and the need to meet schedules, as well as having to keep to a pre-determined route. This causes drivers to get discouraged. Fuel consumption in heavy-duty vehicles depends on a larger set of variables compared to light vehicles [12]. In this case, the context is especially important. The topography, the distance between the stops, the vehicle type, the traffic signs and the road type (urban line or extra-urban line) significantly affect energy consumption.

A pilot test [13] performed with 3 drivers in the city of Rome concluded that driving behavior can reduce fuel consumption by $27 \%$. External variables such as slope can double the fuel consumption and the bus load can also have an impact with increases of up to $26 \%$. Other authors [14] [15] also demonstrated the strong relationship between driving style and fuel consumption.

Educational programs and training tools allow drivers to improve their driving skills. These types of solutions have two main problems: the companies have to pay an efficient driving expert and the driver is not working during the training period. This causes that the duration of the classes is too short. The trainer has to detect in a short time the student's deficiencies. Despite this, the reduction of fuel consumption through training programs might be equal or even higher than if we added technological solutions to the vehicle [16]. Furthermore, these types of solutions avoid that the company has to install new elements in the bus fleet with the consequent expense.

On the other hand, many studies highlight that the improvement in the driving style is degraded in the long term [17]. In [5] the authors conducted a study with urban bus drivers in order to assess the impact of an eco-driving training course on their driving behavior. The results showed a $10.2 \%$ decrease in fuel consumption immediately after the course. This value was reduced to $4.35 \%$ two months after the training phase.

In the literature, many researchers have developed methods for classifying drivers [18] [19] [20] [21]. Classification methods can be divided into two sets: supervised and unsupervised. On the one hand, supervised proposals can predict how a new sample will be labeled using a previously labeled training dataset. This is their main drawback. This solution requires manual labeling. Furthermore, this task can be influenced by subjectivity. On the other hand, the aim of unsupervised classification is to find a function to describe a hidden structure from unlabeled data. The main advantage of these methods is that we do not have to label the training dataset. However, unexpected results may arise if the model does not fully reflect reality.

In [22], the authors propose a method based on a Bayesian classifier to identify the type of driver. This solution was validated by 10 drivers with an overall accuracy of $77 \%$. In [23], the researchers propose using Support Vector Machine (SVM) and K-Means clustering to recognize driving styles. The data are obtained through the vehicle's inertial sensors. In this work, they conclude that turning events and brake events are very useful to differentiate drivers. They also highlight that the number of features analyzed is limited and that others such as driver body posture, head dynamics and hand location may be very useful to analyze the driver. In [24], the authors develop a rule-based approach in order to build a driving style classification. The drawback of this solution is the complexity of modeling more general driving behaviors. This proposal was only validated in a simulator.

In [25], the authors present an algorithm to classify the driver behavior in two types: normal and aggressive driving. This algorithm is based on the Auto Regressive with Exogenous input model (ARX). The model is validated on data recorded on a test track, in a vehicle driven by two different drivers (a 
normal driver and an aggressive driver). The results were that the relevant sensitivity is $90.3 \%$ and the relevant specificity is $88.6 \%$ when they only took into account the driving samples obtained when the road curvature was high. In the other cases, the sensitivity of the model obtained is $50.8 \%$, while the specificity is $91.7 \%$.

Additionally, there are solutions based on the unsupervised classification method. A clustering-based approach is presented in [26]. They use a system for real-time vehicle tracking consisting of a GPS and GSM/GPRS data transmission. This system provides the GPS position, time, and speed values. From the speed values, they calculate the longitudinal acceleration and the mechanical work (the energy required to increase the speed over the time). The data is sent to a central system and then analyzed using two clustering methods: Hierarchical cluster analysis (HCA) and Principal Component Analysis (PCA).

In [27] the authors also use a clustering-based solution to identify different driving behaviors. The algorithm segments and clusters car-following behaviors based on eight variables: the longitudinal acceleration, the lateral acceleration, the yaw rate, the vehicle speed, the lane offset, the yaw angle, the range, and the range rate. The solution is applied to a dataset obtained by 20 different drivers ( 10 car and 10 truck drivers). The results show that each car driver has a personal driving behavior, whereas truck drivers show a common driving pattern.

A quantitative method to evaluate driving styles is presented in [21]. A personalized driver model is established for each driver by using real-world vehicle test conditions and the locally designed neural network. This model is employed to perform the simulated standard driving cycle test for driving behavior normalization, where the desired speed profile is adopted from a standard driving cycle test (FTP-75) which is described in [28]. Finally, they proposed an aggressiveness index based on the energy spectral density analysis on normalized behavior. This index is applied to detect abnormal driving behavior using a simulator. In [29], the researchers present an algorithm to recognize driving behaviors based on the Bayesian Multivariate Linear Model with a sequence segmentation algorithm.

In [30], the researchers propose a method based on sudden accelerations. The algorithm extracts jerk features from the current vehicle speed within a short window, and classifies the current driver style into three classes, calm, normal and aggressive, by comparing the extracted jerk feature with the statistics of the driver styles on the current roadway. The solution is validated using a simulator (PSAT-Powertrain System Analysis Toolkit). They observed that the fuel rate of a conventional vehicle had a positive correlation with spikes in the speed up profile.

In [31], we proposed a solution to evaluate the driving performance based on fuzzy logic. This proposal is used in order to build a gamification tool. The driver obtains points according to his/her driving style. This score allows us to create a ranking. In addition, the system warns the driver when he or she carries out inefficient driving actions. The system was validated in real tests by 36 different drivers on three routes. The results showed an improvement of $8.65 \%$ in fuel consumption when the gamification tool was enabled.
Furthermore, drivers maintain the reduction in the long term. Table 1 shows an overview of the solutions described above.

These proposals described above could be used to inform the trainer in advance about the level of the students. However, these solutions do not indicate what the driver is doing wrong. They are limited to classifying the driver in different classes: calm, normal or aggressive. Other proposals simply assign them a score. In addition, in these solutions we have to previously label driving samples (efficient or inefficient) or calculate bounds. The building of the knowledge database is a laborious task that is done arbitrarily and does not accurately reflect the reality.

The driving style not only depends on the driver, it is also strongly influenced by external factors [12] [13] [32]. Therefore, the result of the evaluation may not be fair. This is particularly important in the case of bus drivers who have to follow fixed routes. Two drivers of different lines cannot be compared directly because the routes differ. One driver may use inertia less than another because the distance between the stops is shorter.

TABLE 1

PROPOSALS TO CLASSIFY DRIVING BEHAVIOR

\begin{tabular}{|c|c|c|c|}
\hline Reference & $\begin{array}{c}\text { Classification } \\
\text { Method }\end{array}$ & Algorithm & Test \\
\hline [22] & Supervised & $\begin{array}{l}\text { Bayesian } \\
\text { Classifier }\end{array}$ & Real \\
\hline [23] & Mixed & $\begin{array}{l}\text { K-Means and } \\
\text { Support Vector } \\
\text { Machine }\end{array}$ & Real \\
\hline [24] & Supervised & Rule-based & Simulator \\
\hline [25] & Supervised & $\begin{array}{c}\text { Auto } \\
\text { Regressive with } \\
\text { Exogenous } \\
\text { Input Model } \\
\end{array}$ & Real \\
\hline [26] & Unsupervised & $\begin{array}{c}\text { Hierarchical } \\
\text { Cluster } \\
\text { Analysis } \\
\text { (HCA) and } \\
\text { Principal } \\
\text { Component } \\
\text { Analysis } \\
\end{array}$ & Real \\
\hline [27] & Unsupervised & Clustering & Real \\
\hline [21] & Unsupervised & $\begin{array}{c}\text { Artificial } \\
\text { Neural } \\
\text { Network } \\
\end{array}$ & Simulator \\
\hline [29] & Unsupervised & $\begin{array}{c}\text { Bayesian } \\
\text { Multivariate } \\
\text { Linear Model }\end{array}$ & Real \\
\hline [30] & Unsupervised & Fuzzy Logic & Real \\
\hline [31] & Unsupervised & Fuzzy Logic & Real \\
\hline
\end{tabular}

Our solution calculates the efficiency comparing with a peer or combination of peers. Therefore, the results are realistic. The system highlights that a driver is less efficient if there is another that has better results under similar conditions. Furthermore, our proposal determines what causes inefficiency and quantifies it. Another advantage is that we can use inputs and outputs with 
different measurement units. This is very useful in this context because the data can be obtained from very different sources.

\section{DESCRIPTION OF THE PROPOSAL}

The vehicle's telemetry is required in order to evaluate the driving. In our case, we use the Cated Box ${ }^{\circledR}$ system [33] [34] developed by ADN Context-aware Mobile Solutions S.L. and our research group. Figure 1 shows the system. This solution is able to obtain vehicle variables such as speed, rpm, longitudinal acceleration, total distance, instantaneous fuel consumption or engine load. The communication protocol that makes the gathering of these data possible is SAE J1939 [35]. In addition, the solution has a GPS to locate the bus. These data are used to detect the driving patterns. Driving patterns are different combinations of events captured along a temporal window. Other proposals are based on single events or their instantaneous combination. These solutions might be more sensitive to events outside the driver's control such as a pedestrian crossing the road incorrectly, causing a harsh deceleration.

The next step is to calculate the driver's performance for each pattern. In this case, we have defined different key performance indicators (KPIs). The driving patterns and the KPIs are saved in a relational database to allow complex queries.

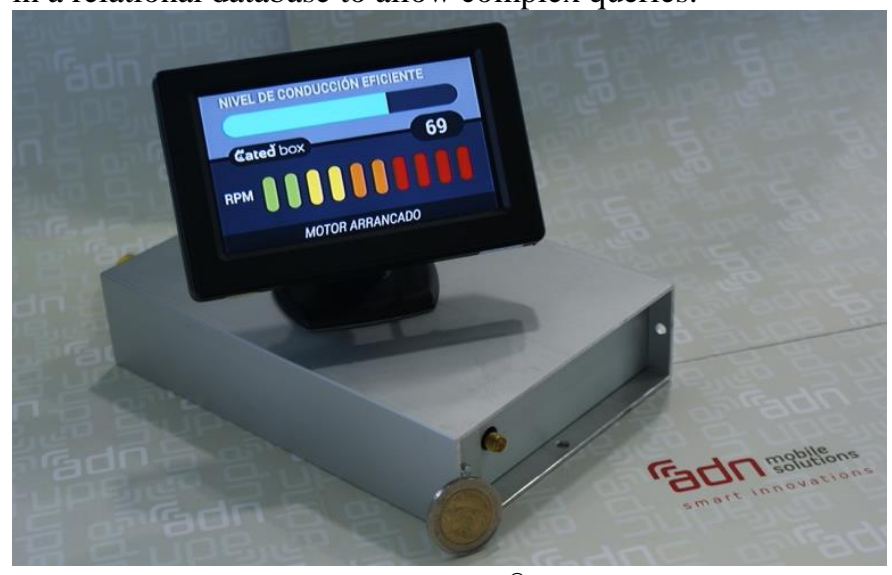

Fig. 1. Cated Box ${ }^{\circledR}$ system.

Finally, we use the KPIs to calculate the efficiency of each driver. The technique used to obtain efficiency is known as Data Envelopment Analysis (DEA). The result is a value between zero and one for each driver where one means the driver is efficient. In addition, this algorithm provides slack values for each driver. These values indicate how much the driver would have to improve a KPI in order to be efficient. This method allows us to ascertain the main causes why a driver does not drive efficiently. The solution is described in more depth in the following subsections.

\section{A. Parameters required to evaluate the driving}

One of the keys to reduce fuel consumption is to minimize energy loss. The first step is to detect the behaviors that have a strong impact on fuel consumption. We have developed driving patterns [36] based on the following proven facts:

- Acceleration causes a very significant increase in energy demand. This action should be made when the driver is really sure that that energy will be taken advantage of.

- There is no tractive force at the wheels when the vehicle is in motion with inertia. We must avoid losing energy due to braking force if this does not imply a problem for safety. The motion of the vehicle should only be limited by the aerodynamic force, the rolling force, and the gravity force in order to save fuel. The opposition of these forces to the movement is inevitable. The mechanical parts of the vehicle also behave as a brake, known as "Engine brake".

- The vehicle consumes fuel when the vehicle's engine is running and the vehicle is not in motion. In this case, the fuel consumption depends on the energy required to keep the engine running and the accessories. Even if the energy demand is small, the fuel consumption might be important if the vehicle is in this state for a long period of time.

TABLE 2

DESCRIPTION OF THE DRIVING PATTERNS

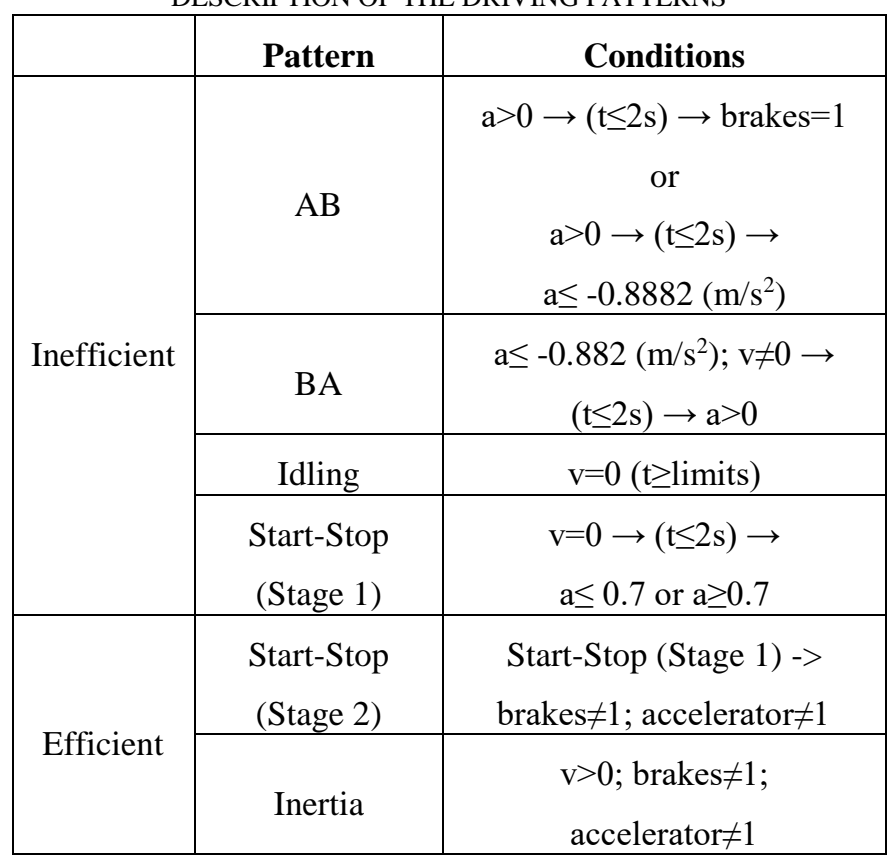

Table 2 describes the driving patterns that we use to evaluate the driving style. The driving patterns can be classified into two groups: efficient patterns and inefficient patterns. We have used the following:

Acceleration-Brake pattern $(\mathrm{AB})$ : it is used to detect situations in which the driver is not taking advantage of inertia. The driver presses the accelerator pedal followed by a deceleration $\left(a \leq-0.882 \mathrm{~m} / \mathrm{s}^{2}\right)$ within a maximum of two seconds. In this case, the energy produced by burning fuel is dissipated as heat through the brakes. The acceleration threshold $\left(-0.882 \mathrm{~m} / \mathrm{s}^{2}\right)$ was calculated by experts of our partner company (ADN Context-aware Mobile Solutions S.L) and our research group after analyzing the telemetry and fuel consumption of more than 1000 buses from different public bus fleets [41]. Frequent occurrence of this pattern during driving 


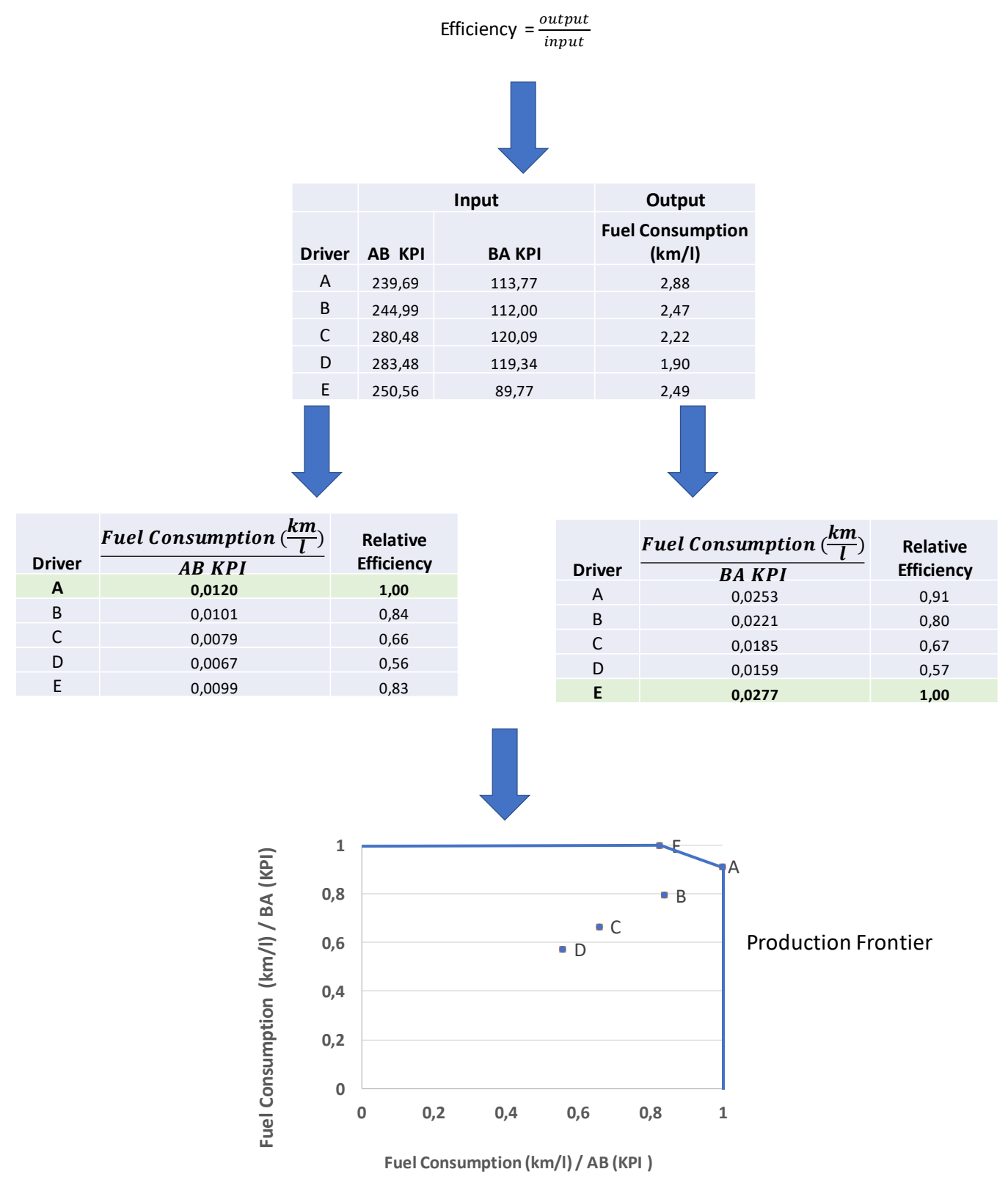

Fig. 2. Example of Data Envelopment Analysis.

indicates that the driver does not anticipate the traffic flow. This is a bad practice for both fuel consumption and safety.

Brake-Acceleration pattern (BA): it is responsible for describing situations in which the driver decelerates $(a \leq$ $-0.882 \mathrm{~m} / \mathrm{s}^{2}$ ) and is followed by an acceleration within a maximum of two seconds, without having stopped the vehicle. This could be indicative of the driver not keeping the safety distance. As with the previous pattern the energy produced by the engine is wasted.

Idling: this pattern occurs when the vehicle is stationary, but the engine is on. If this state is maintained for a long time, the fuel consumption is significantly affected, especially in the case of buses and trucks. Drivers of these vehicles have to drive for a significant period of time. Therefore, it is mandatory that drivers take a rest. During this time, they often keep the engine idling. The result is an increase in pollutant emissions and energy loss [37].

Inertia: when the vehicle is moving using kinetic energy there is no fuel consumption. The tractive force comes only from the accumulated energy. The gravity force and the rolling force will reduce the speed of the vehicle. Modern vehicles use an electronic injection system. This system cuts off the supply of fuel to the engine when it can work by inertia. The clutch must not be used to decouple the engine from the rest of the transmission. If the engine is disconnected from the traction it is necessary to burn fuel to keep it running. This characteristic of the engines allows to save fuel and reduce the brake wear of the vehicle. However, it requires that the driver has the ability to anticipate the traffic flow [38][39]. The use of inertia is a technique widely used in eco-driving [40]. 
Stop-Start: this pattern has two stages. In the first stage, the vehicle is stationary and the driver begins to accelerate. In that case, we assess whether the driver is accelerating correctly. It checks if the acceleration value is near the threshold considered as efficient, which in our case is $0.7 \mathrm{~m} / \mathrm{s}^{2}$. This threshold has been empirically calculated analyzing the driving samples obtained by bus fleets since 2012 [41]. In the second stage, the driver must maximize the time that he or she is driving using inertia. This stage starts when the driver stops accelerating. Our proposal positively evaluates that the driver uses inertia as the fuel consumption is zero.

In order to measure the performance level of the driver for each driving pattern we have defined Key Performance Indicators (KPIs). The set of KPIs considered are described in Table 3.

TABLE 3

DESCRIPTION OF THE KPIS

\begin{tabular}{|c|c|}
\hline KPI & Conditions \\
\hline AB & $\begin{array}{c}\text { Number of AB pattern occurrences per } \\
100 \mathrm{~km} .\end{array}$ \\
\hline BA & $\begin{array}{c}\text { Number of BA pattern occurrences per } \\
100 \mathrm{~km} .\end{array}$ \\
\hline Idling & $\begin{array}{c}\text { Time percentage spent idling in relation } \\
\text { to the total duration of the route. }\end{array}$ \\
\hline $\begin{array}{c}\text { Start-Stop } \\
\text { (Stage 1) }\end{array}$ & $\begin{array}{c}\text { Difference between the ideal acceleration } \\
\text { and the actual acceleration of the vehicle } \\
\text { expressed as a percentage when the } \\
\text { vehicle is set in motion. }\end{array}$ \\
\hline $\begin{array}{c}\text { Start-Stop } \\
\text { (Stage 2) }\end{array}$ & $\begin{array}{c}\text { Percentage of times not driving in inertia } \\
\text { after the first stage of Start-Stop pattern. }\end{array}$ \\
\hline Non-Inertia & $\begin{array}{c}\text { Time percentage not driving with inertia } \\
\text { in relation to the total duration of the } \\
\text { route }\end{array}$ \\
\hline
\end{tabular}

\section{B. Evaluating efficient driving}

We use Data Envelopment Analysis (DEA) to assess the driving efficiency. It is a non-parametric method to provide a relative efficiency assessment (called DEA efficient) for a group of decision-making units (DMU) or for productive efficiency (aka technical efficiency) with a multiple number of inputs and outputs. DMUs are the elements whose efficiency we are going to evaluate. In this paper, each DMU represents a different bus driver.

DEA was first proposed in 1978 [42] and is commonly used in operations research and economics to empirically measure productive efficiency of DMUs. In order to determine whether a DMU is efficient is as easy as checking if the DMU is on the "frontier" of the production possibility set. In this way, DEA identifies a "frontier" on which the relative performance of all the utilities in the sample can be compared.
Figure 2 captures how Data Envelopment Analysis works. This technique compares the relative efficiency of DMUs when they perform similar tasks. In this example, the DMUs are the different bus drivers. They utilize similar resources to generate similar outputs. In order to calculate the efficiency, it is defined as output/input.

In this case, the inputs are the AB KPI (the number of times per $100 \mathrm{~km}$ that the driver accelerates and then decelerates) and the BA KPI (the number of times per $100 \mathrm{~km}$ that the driver decelerates and then accelerate). In both cases, there is not an inertia period. The output is the fuel consumption $(\mathrm{km} / \mathrm{l})$. Therefore, we have two ratios that we have to optimize: Fuel Consumption-AB branch and Fuel Consumption-BA.

Observing Figure 2, we can see that the highest ratio of Fuel Consumption- $\mathrm{AB}$ is $\mathrm{A}$. However, the highest ratio Fuel Consumption -BA is E. These two DMUs define the production frontier. The positions on the graph of drivers $\mathrm{A}$ and $\mathrm{E}$ show a performance level which is superior to all the other DMUs. The efficiency frontier 'envelops' the inefficient DMUs. Any DMU on the frontier is considered efficient (the value is 1). As a result, it can be used as a threshold against which to measure the performance.

There are two different DEA models: variable returns to scale (VRS) and constant returns to scale (CRS). On the one hand, VRS suggests that an increase in the amount of inputs utilized can result in a proportional or non-proportional change in the amount of outputs generated. On the other hand, the CRS model implies that an increase in the amount of inputs utilized causes a proportional increase in the amount of outputs generated. VRS is a more realistic model.

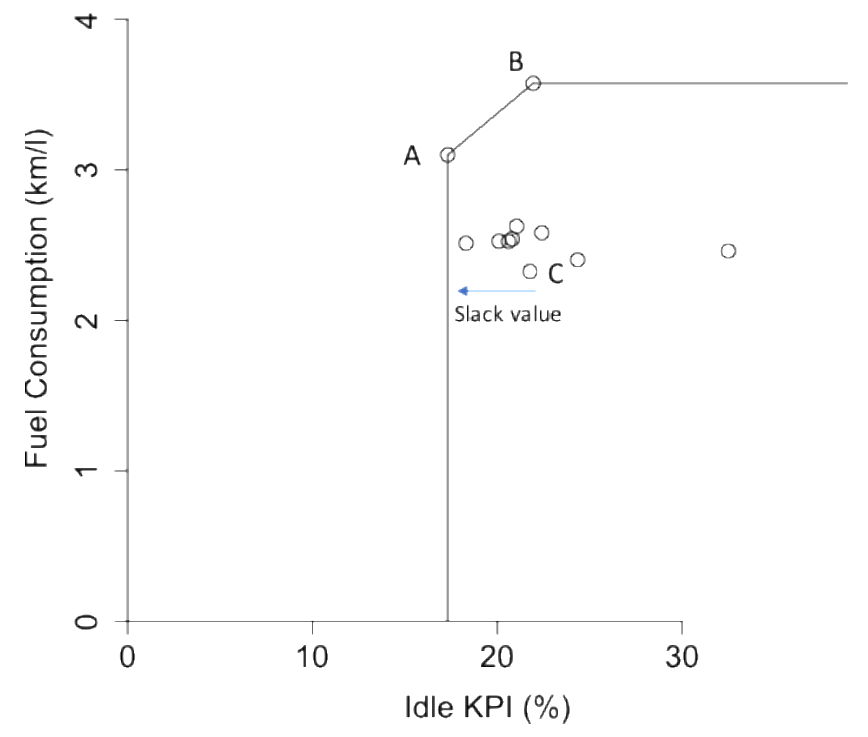

Fig. 3. Example of DEA frontier using VRS (variable return scale).

Furthermore, DEA has three different orientations: input oriented, output oriented, and input-output oriented. Each of these orientations assumes that we are capable of modifying inputs, outputs or inputs and outputs respectively. In our case, fuel consumption is used as output because it does not only 
depend on driving style. Fuel consumption is influenced by many variables foreign to the driver such as the number of passengers, weather conditions or state of the traffic [12] [13]. For this reason, it is not always the best way to evaluate the driving style. Our proposal is focused on detecting the incorrect driving behaviors. We employ the input-oriented model of Data Envelopment Analysis (DEA). In this model, fuel consumption remains fixed and the aim is to minimize the inputs.

In order to evaluate the driving, we have used an inputoriented model and variable returns to scale (VRS). In this case an improvement in the inputs is not translated directly into a proportional improvement in the output. The energy consumption of the vehicles depends on many factors, some of which are beyond the control of the driver. However, many research papers [43] show that eco-driving reduces fuel consumption, although the improvement is very variable depending on the situation.

Figure 3 shows an example of a production frontier in an input-oriented DEA model using VRS for idle KPI. The data were obtained from a real bus line in Asturias (Spain). In this case, the DMUs A and B are efficient when forming part of the border. On the contrary, $\mathrm{C}$ is inefficient. The distance between $\mathrm{C}$ and the border on the $\mathrm{X}$-axis (slack value) is what this driver should improve in order to be efficient.

In our proposal, the objective is to obtain the efficiency of each user. Drivers whose efficiency is higher are those who act as models for the rest of the drivers. If we consider a set of $n$ drivers (DMUs), where $\mathrm{DMU}_{\mathrm{k}} \mathrm{k}=1, \ldots, \mathrm{n}$, the efficiency measure $h_{k}$ for $\mathrm{DMU}_{\mathrm{k}}$ is calculated by solving the following equation:

$$
\text { Maximize } h_{k}=\frac{\sum_{r=1}^{S} u_{r} y_{r k}}{\sum_{i=1}^{m} v_{i} x_{i k}}
$$

Subject to:

$$
\begin{gathered}
\frac{\sum_{r=1}^{s} u_{r} y_{j}}{\sum_{i=1}^{m} v_{i} x_{j}} \leq 1, j=1,2, \ldots, j_{k}, \ldots, n \\
u_{r} \geq \varepsilon, r=1,2, \ldots, s \\
v_{i} \geq \varepsilon, i=1,2, \ldots, m
\end{gathered}
$$

Where:

$u_{r}$ and $v_{i}$ are the weights to be determined for output $r$ and input $i$ respectively, $h_{k}$ is the relative efficiency of $\mathrm{DMU}_{\mathrm{k}}, s$ is the number of outputs, $m$ is the number of inputs, $\varepsilon$ is a small positive value (typically of the order of $10^{-5}$ or $10^{-6}$ ), and $n$ is the number of drivers (decision making units).

The relative efficiency of a driver (DMU in DEA) is defined as a ratio of the weighted sums of their outputs (virtual output) and the weighted sums of their inputs (virtual input). Equation 1 shows that the relative efficiency of a driver is always a value between 0 and 1 . The weights $u_{r}$ and $v_{i}$ are determined in the model so that each DMU is as efficient as possible. This avoids us the difficult task of assigning suitable weights to each input and output factor. However, we can add restrictions to the weights if necessary [44].

As we can see in the model, the selection of input and output variables has a strong effect on the behavior of the algorithm.
In this work, we are using an input-oriented model. Therefore, the input variables are controlled by the drivers. However, the output variables cannot be adjusted directly by the driver. We have chosen the input-orientation model because fuel consumption depends on many variables that are out of the driver's control [45].

On the one hand, a driver is considered efficient when Max $h_{k}$ is 1 . On the other hand, a driver is relatively inefficient if it is possible to minimize any of its inputs without decreasing any output and without increasing any other inputs. However, an efficiency value approaching 1 means that the scope for improvement is small. The trainer should focus on the drivers who obtained the scores closest to zero.

The DEA model in equation 1 is nonlinear. [46] propose a transformation to solve it. They fixed the denominator to a constant (unity) in order to change the model to an equivalent linear form. In this case, the aim is to maximize the numerator.

$$
\text { Maximize } h_{k}=\sum_{r=1}^{s} u_{r} y_{r k}
$$

Subject to:

$$
\begin{gathered}
\sum_{i=1}^{m} v_{i} x_{i k}=1 \\
\sum_{r=1}^{s} u_{r} y_{r j}-\sum_{i=1}^{m} v_{i} x_{i j} \leq 0, \quad j=1, \ldots, n \\
u_{r} \geq \varepsilon, r=1,2, \ldots, s \\
v_{i} \geq \varepsilon, i=1,2, \ldots, m
\end{gathered}
$$

Equation 3 shows a dual version of the previous model. It is built by assigning a variable to each constraint in the model described in equation 2. Dual form has fewer constraints. The difficult of solving a problem in linear programming is related to the number of constraints. In general, the more constraints, the more difficult a problem is to solve. For this reason, dual form is computationally simpler and it is used to make the evaluation of driving style.

$$
\text { minimize } Z_{k}-\varepsilon \sum_{r=1}^{s} s_{r}^{+}-\varepsilon \sum_{i=1}^{m} s_{i}^{-}
$$

Subject to:

$$
\begin{gathered}
\sum_{j=1}^{n} \lambda_{j} y_{r j}-s_{r}^{+}=y_{r k}, r=1, \ldots, s \\
Z_{k} x_{i k}-\sum_{j=1}^{n} \lambda_{j} x_{i j}-s_{i}^{-}=0, i=1, \ldots, m \\
\sum_{j=1}^{n} \lambda_{j}=1 \\
s_{r}^{+}, s_{i}^{-} \geq 0 j=1,2, \ldots, n .
\end{gathered}
$$

This model tries to build for each DMU a unit that overtakes it. The composite unit generates an output that is equal to or 
higher than the corresponding output of the original DMU. It uses a proportion $\left(\mathrm{Z}_{\mathrm{k}}\right)$ of the inputs as a maximum. In this way it is possible to obtain the minimum amount by which the inputs must be proportionally reduced to make the DMU efficient. Sometimes, the proportional reduction applied to the inputs is not sufficient. In this case, we should decrease the inputs. The proportion of each input $\left(s_{i}^{-}\right)$that we need to reduce is called slack. $\sum_{j=1}^{n} \lambda_{j}=1$ constraint is included in order to allow variable returns to scale [47]".

We use the KPIs described above as input variables. The objective is to measure the performance of the drivers in each pattern.

Input Variables:

- Acceleration-Brake KPI (number of events per 100 $\mathrm{Km})$

- Brake-Acceleration KPI (number of events per 100 $\mathrm{Km})$

- $\quad$ Idling KPI (\%)

- $\quad$ Stop-Start-Phase 1 KPI (\%)

- $\quad$ Stop-Start-Phase 2 KPI (\%)

- $\quad$ Non-Inertia KPI (\%)

Output Variables:

- Fuel consumption $(\mathrm{Km} / \mathrm{l})$

\section{EXPERIMENTAL EVALUATION}

\section{A. Experimental Setting}

The proposal was validated using data from real tests. The bus was the same in all cases, a Mercedes-Benz Citaro. We have analyzed the driving behavior in two different urban transport lines of Asturias (Spain): route A (Figure 4, the bus line is represented in blue) and route B (Figure 5, the bus line is represented in blue). The length of Route $\mathrm{A}$ is $14.83 \mathrm{Km}$. The average trip time was 46 minutes and 6 seconds. The length of Route B is $10.6 \mathrm{Km}$. In that case, the average trip time was 28 minutes and 40 seconds.

For this study we observed 32 drivers (route A) and 11 drivers (route B). The number of drivers differs on both routes because only some drivers on route $\mathrm{A}$ are also responsible for route $B$. The drivers were between 38 and 60 years of age. The average age was 49 . All the participants are professional drivers and have previous knowledge about efficient driving. Each participant completed the route four times.

The drivers were the workers of the public bus company that manages both routes, A and B. They had served in the company between 2 and 44 years (14 on average). The driving samples were captured between 10:00 a.m. to 13:00 p.m. and 16 p.m. to 18 p.m. from Monday to Friday on dry days in January 2017. The driving tests were carried out during their normal working day. They had no prior knowledge about the study. Table 4 shows driving time for both routes.

Data Envelopment Analysis (DEA) works well if the samples have been captured under similar conditions. To take into account the external factors, we must filter and cluster the data before using DEA. In our case, the driving samples have been clustered according to the route in order to avoid that variables such as the slope, intersections or road curves affect the results. Furthermore, we consider the traffic conditions and the weather. For this reason, the driving tests were made in the time interval indicated before and when it was not raining. In this time interval the traffic is not heavy as most people are at work.

TABLE 4

DRIVING TIME OBTAINED IN THE DRIVING TESTS

\begin{tabular}{ccc}
\hline \hline Variable & Route A & Route B \\
\hline Average Time Driving (minutes) & 49,70 & 29,86 \\
Median Value (minutes) & 50,09 & 29,97 \\
Maximum Time Driving (minutes) & 52,09 & 29,99 \\
Minimum Time Driving (minutes) & 46,46 & 27,03 \\
Standard Deviation of Time Driving & 1,59 & 0,57 \\
(minutes) & & \\
\hline \hline
\end{tabular}

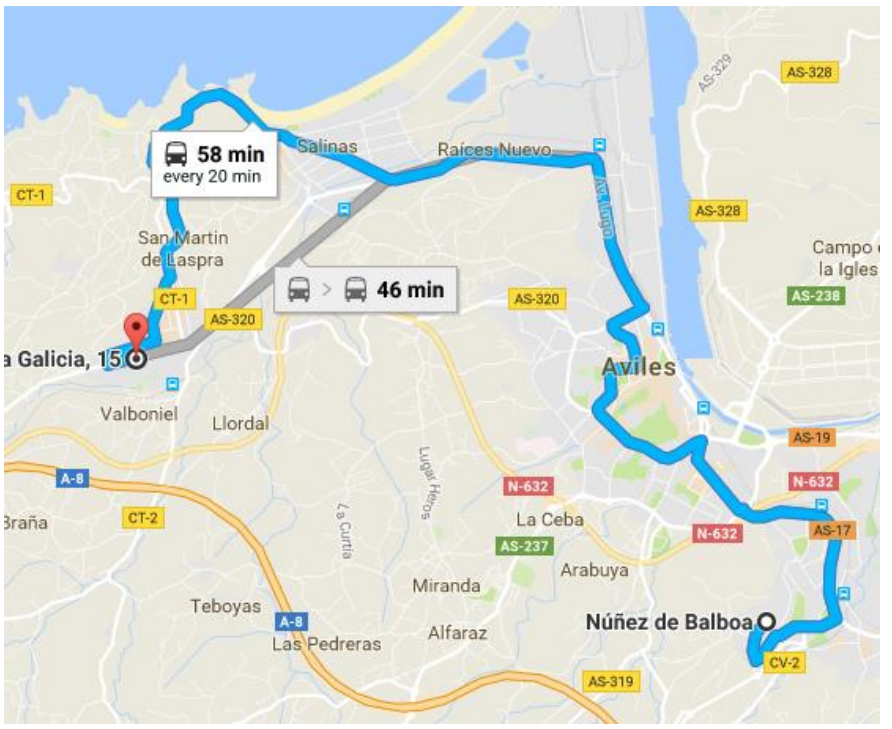

Fig. 4. Route A used to conduct the driving tests.

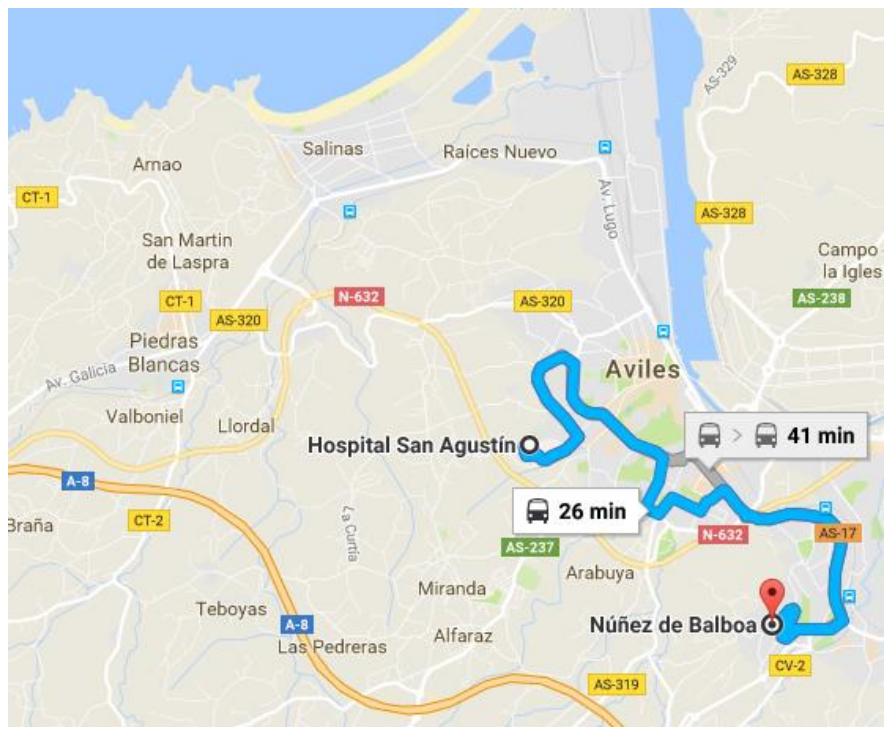

Fig. 5. Route B used to conduct the driving tests. 


\section{B. Results}

Figure 6 captures the efficiency score calculated using DEA for each driver on route A. $46.88 \%$ of the drivers obtained the highest possible score (1). However, there are 17 drivers who can still improve their driving style, but we can see that all drivers got a high score (higher than 0.87).

Figure 7 compares the fuel consumption of the drivers classified as efficient (DEA output $=1$ ) with the less efficient drivers (DEA output $<1$ ) on route A. Fuel consumption is not a fair method to evaluate drivers due to the influence of external factors. Also, it does not provide information to the trainer. However, there is a relationship between driving style and fuel consumption. The eco-friendly driver will always achieve the lowest possible fuel consumption under the current environmental conditions. In Figure 7, we can see how efficient drivers consumed $4.6 \%$ less than the other less efficient drivers. These results show that low values in driving KPIs are closely related to a reduction in fuel consumption.

Table 5 captures the slack values for each variable on route A. These values indicate how much the drivers would have to improve a specific pattern in order to approach the value considered as efficient. If the value obtained is 0 , it means that the drivers cannot further reduce the input value.

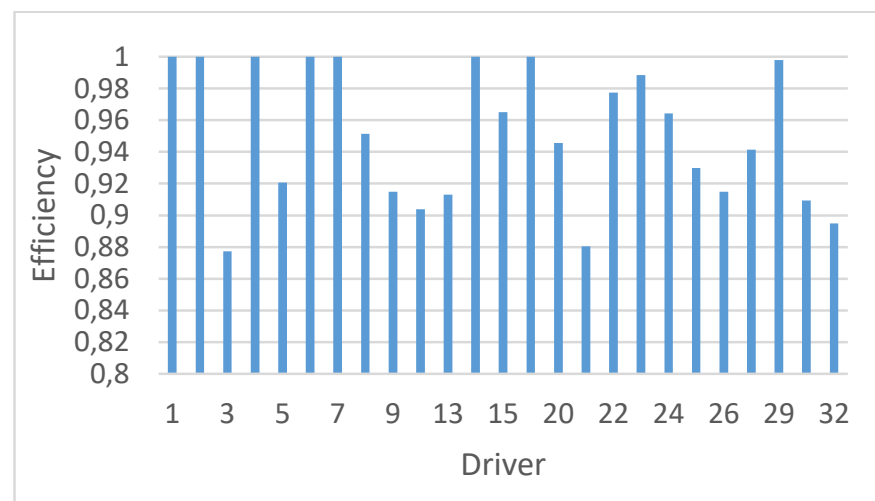

Fig. 6. Efficiency values on route A.

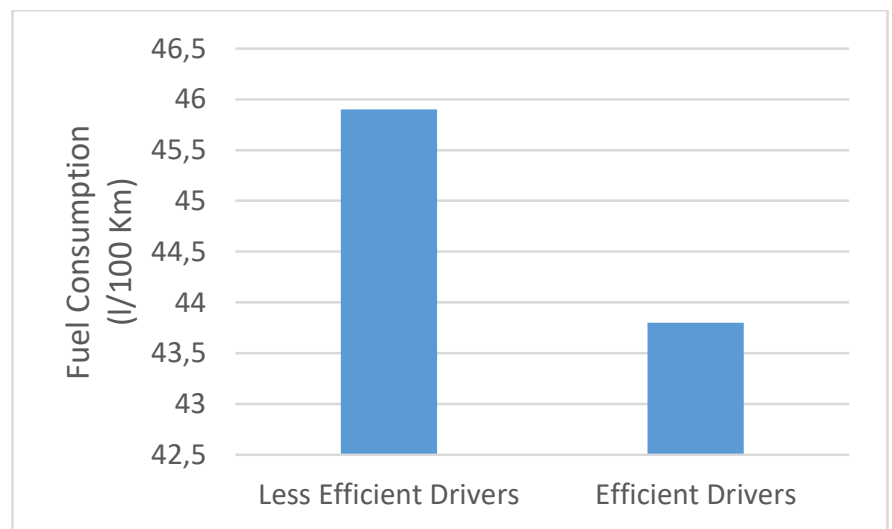

Fig. 7. Comparison of fuel consumption between the efficient drivers and the less efficient drivers on route $\mathrm{A}$.
TABLE 5

SLACK VALUES FOR INPUT VARIABLES ON ROUTE A

\begin{tabular}{cc}
\hline \hline Pattern & Value \\
\hline Acceleration-Brake (Events/100 Km) & 25,34 \\
Brake-Acceleration (Events/100 Km) & 21,66 \\
Idle (\%) & 1,93 \\
Start-Engine_Phase1 (\%) & 8,45 \\
Start-Engine_Phase 2 (\%) & 5,26 \\
Driving without inertia (\%) & 0,00 \\
\hline \hline
\end{tabular}

As the optimal values are calculated based on the results obtained by other bus drivers under similar conditions, we ensure that the marked target is realistic. In this way, we can evaluate the driving without manually fixing the permissible statistical values for each pattern.

On the analyzed route, we can see that the drivers should try to improve above all the acceleration-brake pattern and the brake-acceleration pattern. Drivers show poor ability when they have to anticipate the traffic flow. In addition, it is common that the vehicle is idling during the driver's resting time.

However, the time driving in inertia has little room for improvement. Buses move on urban roads. In this scenario, the traffic signs and bus stops make it difficult for drivers to take advantage of inertia to reduce energy consumption. This does not mean that this driving pattern is not important to save fuel, but in this case all drivers present similar results. Therefore, the possibility of improving is small according to the algorithm.

Figure 8 captures the efficiency score obtained by the drivers on route B. $81.81 \%$ of the drivers were efficient (score $=1$ ). Only two drivers presented a value less than 1 (maximum efficiency) and it was never less than 0.9. Figure 9 shows the fuel consumption obtained by the efficient and less efficient drivers on Route $\mathrm{B}$. The results are better than on route $\mathrm{A}$, despite being a similar scenario as the route is in the same city. This is reflected in the efficiency obtained by the different drivers on Route B.

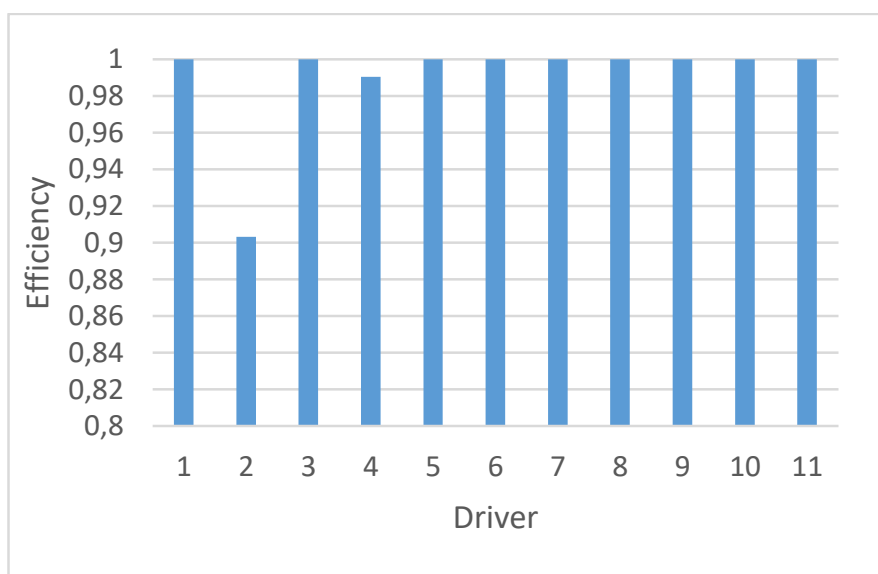

Fig. 8. Efficiency values on Route B 


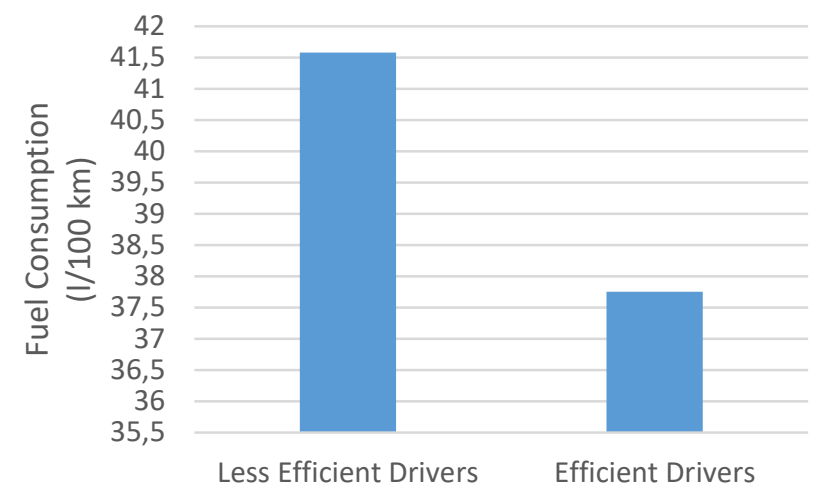

Fig. 9. Comparison of fuel consumption between efficient drivers and less efficient drivers.

Table 6 captures the slack values for each KPI on route B. As with route A, drivers should improve above all the acceleration-brake KPI and the brake-acceleration KPI. However, all participants on this route have very similar values and the improvement margin is not as wide as on route $\mathrm{A}$.

TABLE 6

SLACK VALUES FOR INPUT VARIABLES ON ROUTE B

\begin{tabular}{cc}
\hline \hline Pattern & Value \\
\hline Acceleration-Brake (Events/100 Km) & 5,72 \\
Brake-Acceleration (Events/100 Km) & 5,96 \\
Idling (\%) & 0,05 \\
Start-Engine_Phase 1 (\%) & 2,06 \\
Start-Engine_Phase 2(\%) & 0,65 \\
Driving without inertia $(\%)$ & 0 \\
\hline \hline
\end{tabular}

Figure 10 shows a comparison of the results obtained on routes A and B by drivers who drove on both. We can observe that they present similar behavior in the two scenarios. The driver with the most different behavior is driver 4 . In this case, the efficiency was reduced by $7 \%$ on route $\mathrm{A}$ with respect to the value obtained on route B. On average, drivers worsen $1.33 \%$ on route $\mathrm{A}$ in comparison with the efficiency values they obtain on route $\mathrm{B}$.

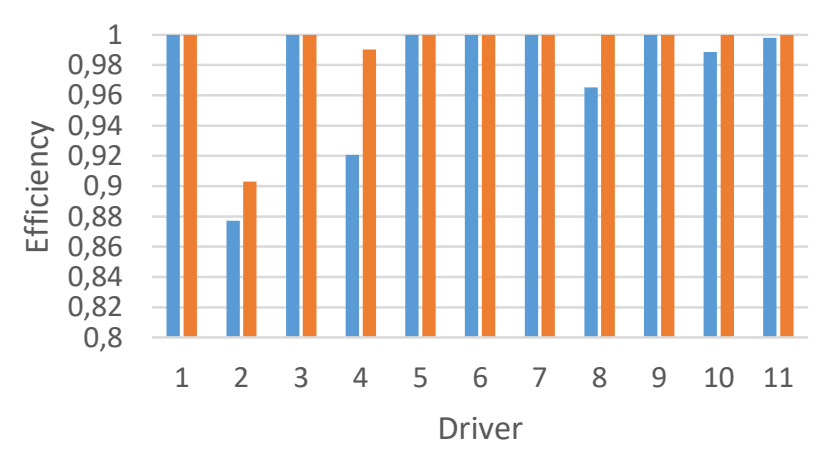

Route A $\square$ Route B

Fig. 10. Comparison of efficiency values obtained by drivers who drove on both routes.

\section{CONCLUSION}

In this paper, we have proposed a method to objectively evaluate the driving of bus drivers. The solution is based on data analysis envelopment (DEA) and the analysis of driving behavior in order to detect in what areas the driver should improve in order to reduce fuel consumption.

The main strength of this proposal is that it is strongly adjusted to reality. Ideal acceleration or deceleration values are very difficult to achieve due to external factors. For this reason, inflexible evaluation systems discourage drivers. For example, if a tool requires the driver to drive $50 \%$ of the time using inertia in order to be classified as efficient, but there are many traffic signs that make this aim impossible for the driver, he or she will be frustrated and will disable the system.

Another advantage of this proposal based on DEA is that it allows us to determine which are the behaviors that the driver must correct in order to take advantage of the energy generated by the engine. This is possible because the DEA model builds a production frontier. This frontier is generated by the set of efficient drivers. Therefore, we can calculate the proportion of each input that we need to reduce in order to obtain a production ratio (output / inputs) equal to that obtained by efficient drivers.

We have used the proposal to analyze the driving performance on two bus lines in Spain. The results showed that in both cases all drivers have good skills in driving efficiency. All participants achieved a score higher than 0.8 (the scale is between 0 and 1). However, we also observed that many of them could improve patterns related to traffic anticipation, which is the main factor to consider in order to drive efficiently. The efficient driving expert should focus on showing the driver how to correctly process the environment information in order to incorporate anticipation into his or her decisions.

The present method is suitable to evaluate the drivers and establish reward plans to encourage drivers to improve their driving. However, we must be very careful when designing these plans to avoid confrontation between participants. The method also allows companies to develop improvement proposals that fit the driving profiles of their employees.

The limitation of this proposal is that drivers would have to follow the same route and under the same environmental conditions in order to make a fair comparison. This requirement may be overcome by introducing a previous stage where the driving samples with similar characteristics are clustered taking into account the vehicle and road type, the geometry of the road, and the environmental conditions (weather, traffic and complexity of the traffic signs). In this study, we have taken into account the traffic, the geometry of the road, and weather conditions. For this reason, we have compared drivers who completed the same route and in similar conditions (driving from Monday to Friday between 10:00-13:00 a.m. and 16:0018:00 p.m. and without rain).

As future work, we want to analyze if comfort influences efficiency. For example, we might compare driving samples captured under different temperatures in the cockpit or analyze the air quality inside the vehicle. Furthermore, we aim to develop a driving assistant that minimizes the appearance of inefficient patterns. The idea is that the assistant recommends 
an appropriate speed and provides information about the environment in order to avoid energy loss when braking. Other future lines of study could be the relationship between driving patterns, the stress of drivers and passenger safety, as all these issues are strongly related.

\section{REFERENCES}

[1]R. T. Burnett et al., "An Integrated Risk Function for Estimating the Global Burden of Disease Attributable to Ambient Fine Particulate Matter Exposure," Environmental Health Perspectives, Feb. 2014.

[2] "Air Quality in Europe 2017," European Environment Agency. [Online]. Available: https://www.eea.europa.eu/publications/air-quality-in-europe-2017. [Accessed: 29-Apr-2018].

[3]S. Henschel, "Health risks of air pollution in Europe - HRAPIE project," $p$. 65.

[4]F. Caiazzo, A. Ashok, I. A. Waitz, S. H. L. Yim, and S. R. H. Barrett, “Air pollution and early deaths in the United States. Part I: Quantifying the impact of major sectors in 2005," Atmospheric Environment, vol. 79, pp. 198-208, Nov. 2013

[5]M. Zarkadoula, G. Zoidis, and E. Tritopoulou, "Training urban bus drivers to promote smart driving: A note on a Greek eco-driving pilot program," Transportation Research Part D: Transport and Environment, vol. 12, no. 6, pp. $449-451$, Aug. 2007

[6]K. Ayyildiz, F. Cavallaro, S. Nocera, and R. Willenbrock, "Reducing fuel consumption and carbon emissions through eco-drive training," Transportation Research Part F: Traffic Psychology and Behaviour, vol. 46, pp. 96-110, Apr. 2017.

[7]A. E. af Wåhlberg, "Long-term effects of training in economical driving: Fuel consumption, accidents, driver acceleration behavior and technical feedback," International Journal of Industrial Ergonomics, vol. 37, no. 4, pp. 333-343, Apr. 2007

[8]L. Pozueco et al., "A methodology to evaluate driving efficiency for professional drivers based on a maturity model," Transportation Research Part C: Emerging Technologies, vol. 85, pp. 148-167, Dec. 2017.

[9] H. Najadat and I. Alsmadi, "An Interactive DEA Based Management System for Hospitals," in Proceedings of the International MultiConference of Engineers and Computer Scientists 2011, Hong Kong, 2011, vol. 2, p. 5.

[10] C. T. Kuah and K. Y. Wong, "Efficiency assessment of universities through data envelopment analysis," Procedia Computer Science, vol. 3, pp. 499-506, 2011.

[11] H. K. Strömberg and I. C. M. Karlsson, "Comparative effects of ecodriving initiatives aimed at urban bus drivers - Results from a field trial," Transportation Research Part D: Transport and Environment, vol. 22, pp. 2833, Jul. 2013

[12] C. Rolim, P. Baptista, G. Duarte, T. Farias, and J. Pereira, "Real-Time Feedback Impacts on Eco-Driving Behavior and Influential Variables in Fuel Consumption in a Lisbon Urban Bus Operator," IEEE Transactions on Intelligent Transportation Systems, vol. 18, no. 11, pp. 3061-3071, Nov. 2017. [13] S. Carrese, A. Gemma, and S. La Spada, "Impacts of Driving Behaviours, Slope and Vehicle Load Factor on Bus Fuel Consumption and Emissions: A Real Case Study in the City of Rome," Procedia - Social and Behavioral Sciences, vol. 87, pp. 211-221, Oct. 2013.

[14] M. J. M. Sullman, L. Dorn, and P. Niemi, "Eco-driving training of professional bus drivers - Does it work?," Transportation Research Part C: Emerging Technologies, vol. 58, pp. 749-759, Sep. 2015.

[15] J. Van Mierlo, G. Maggetto, E. Van de Burgwal, and R. Gense, "Driving style and traffic measures-influence on vehicle emissions and fuel consumption," Proceedings of the Institution of Mechanical Engineers, Part D: Journal of Automobile Engineering, vol. 218, no. 1, pp. 43-50, Jan. 2004

[16] "Energy Efficiency Measures for Buses and Bus Transport—Possibilities and Experiences From Other Actors," Sweden, 2012.

[17] J. S. Hickman, R. J. Hanowski, and O. Ajayi, "Evaluation of an Onboard Safety Monitoring Device in Commercial Vehicle Operations," presented at the International Driving Symposium on Human Factors in Driver Assessment, Training and Vehicle Design, 2009, pp. 38-45.

[18] S. M. Hegazy and M. N. Moustafa, "Classifying aggressive drivers for better traffic signal control," in 2017 IEEE 20th International Conference on Intelligent Transportation Systems (ITSC), 2017, pp. 702-707.

[19] N. Somsuk, P. Pongpanich, and S. Teekasap, "Determining and classifying drivers of sustainable competitive advantages in green supply chain management: Resource-based and relational views," in 2013 IEEE International Conference on Industrial Engineering and Engineering
Management, 2013, pp. 655-660

[20] W. Wang, J. Xi, A. Chong, and L. Li, "Driving Style Classification Using a Semisupervised Support Vector Machine," IEEE Transactions on HumanMachine Systems, vol. 47, no. 5, pp. 650-660, Oct. 2017.

[21] B. Shi et al., "Evaluating Driving Styles by Normalizing Driving Behavior Based on Personalized Driver Modeling," IEEE Transactions on Systems, Man, and Cybernetics: Systems, vol. 45, no. 12, pp. 1502-1508, Dec. 2015.

[22] G. Kedar-Dongarkar and M. Das, "Driver Classification for Optimization of Energy Usage in a Vehicle," Procedia Computer Science, vol. 8, pp. 388393, 2012.

[23] M. V. Ly, S. Martin, and M. M. Trivedi, "Driver classification and driving style recognition using inertial sensors," in 2013 IEEE Intelligent Vehicles Symposium (IV), Gold Coast, 2013, pp. 1040-1045.

[24] C.-C. Lin, S. Jeon, H. Peng, and J. M. Lee, "Driving Pattern Recognition for Control of Hybrid Electric Trucks," Vehicle System Dynamics, vol. 42, no. $1-2$, pp. 41-58, Dec. 2004.

[25] M. Sundbom, P. Falcone, and J. Sjöberg, "Online driver behavior classification using probabilistic ARX models," in 16th International IEEE Conference on Intelligent Transportation Systems (ITSC 2013), 2013, pp. $1107-1112$

[26] Z. Constantinescu, C. Marinoiu, and M. Vladoiu, "Driving Style Analysis Using Data Mining Techniques," International Journal of Computers Communications \& Control, vol. 5, no. 5, pp. 654-663, Dec. 2010.

[27] B. Higgs and M. Abbas, "Segmentation and Clustering of Car-Following Behavior: Recognition of Driving Patterns," IEEE Transactions on Intelligent Transportation Systems, vol. 16, no. 1, pp. 81-90, Feb. 2015.

[28] W. F. Milliken and D. L. Milliken, Race Car Vehicle Dynamics. SAE International, 1995.

[29] A. Bender, G. Agamennoni, J. R. Ward, S. Worrall, and E. M. Nebot, "An Unsupervised Approach for Inferring Driver Behavior From Naturalistic Driving Data," IEEE Transactions on Intelligent Transportation Systems, vol. 16, no. 6, pp. 3325-3336, Dec. 2015.

[30] Y. L. Murphey, R. Milton, and L. Kiliaris, "Driver's style classification using jerk analysis," in 2009 IEEE Workshop on Computational Intelligence in Vehicles and Vehicular Systems, 2009, pp. 23-28.

[31] A. R. Rodríguez, D. M. Álvarez, X. G. Pañeda, D. A. Carbajal, J. E. Jiménez, and F. F. Linera, "Tutoring System for the Efficient Driving of Combustion Vehicles," IEEE Revista Iberoamericana de Tecnologias del Aprendizaje, vol. 8, no. 2, pp. 82-89, May 2013.

[32] R. Lancaster, R. Ward, Great Britain, and Health and Safety Executive, The contribution of individual factors to driving behaviour: implications for managing work-related road safety. United Kingdom: H.M.S.O., 2002.

[33] A. Rionda et al., "UrVAMM \#×2014; A full service for environmentalurban and driving monitoring of professional fleets," in 2013 International Conference on New Concepts in Smart Cities: Fostering Public and Private Alliances (SmartMILE), 2013, pp. 1-6.

[34] A. G. Pañeda et al., "An Architecture for a Learning Analytics System Applied to Efficient Driving," IEEE Revista Iberoamericana de Tecnologias del Aprendizaje, vol. 11, no. 3, pp. 137-145, Aug. 2016.

[35] J. SAE, "Recommended Practice for a Serial Control and Communications Vehicle Network," SAE J1939 Standards Collection, 2007.

[36] L. Pozueco et al., "Adaptive learning for efficient driving in urban public transport," in 2015 International Conference on Computer, Information and Telecommunication Systems (CITS), 2015, pp. 1-5.

[37] S. M. A. Rahman, H. H. Masjuki, M. A. Kalam, M. J. Abedin, A. Sanjid, and H. Sajjad, "Impact of idling on fuel consumption and exhaust emissions and available idle-reduction technologies for diesel vehicles - A review," Energy Conversion and Management, vol. 74, pp. 171-182, Oct. 2013.

[38] C. Silva, M. Ross, and T. Farias, "Analysis and simulation of 'low-cost' strategies to reduce fuel consumption and emissions in conventional gasoline light-duty vehicles," Energy Conversion and Management, vol. 50, no. 2, pp. 215-222, Feb. 2009.

[39] "When Coasting to a Stop, do Not Shift Into Neutral," Car and Driver. [Online]. Available: http://www.caranddriver.com/features/gas-pains-mileagemyths-and-misconceptions. [Accessed: 03-May-2018].

[40] J. Lee, D. J. Nelson, and H. Lohse-Busch, "Vehicle Inertia Impact on Fuel Consumption of Conventional and Hybrid Electric Vehicles Using Acceleration and Coast Driving Strategy," presented at the SAE World Congress \& Exhibition, Warrendale, PA, 2009.

[41] A. Rionda et al., "Blended learning system for efficient professional driving," Computers \& Education, vol. 78, pp. 124-139, Sep. 2014.

[42] A. Charnes, W. . Cooper, B. Golany, L. Seiford, and J. Stutz, "Foundations of data envelopment analysis for Pareto-Koopmans efficient empirical production functions," Journal of Econometrics, vol. 30, no. 1-2, pp. 
91-107, Feb. 1985.

[43] H. Yang and W.-L. Jin, "A control theoretic formulation ofgreen driving strategies based on inter-vehicle communications," Transportation Research Part C: Emerging Technologies, vol. 41, pp. 48-60, Apr. 2014.

[44] A. Charnes, W. W. Cooper, Z. M. Huang, and D. B. Sun, "Polyhedral Cone-Ratio DBA Models with an Illustrative Application to Large Commercial Banks," Journal of Econometrics, vol. 46, pp. 73-91, Feb. 1990.

[45] M. S. Campos, A. Fernández-Montes, J. M. Gavilan, and F. Velasco, "Public resource usage in health systems: a data envelopment analysis of the efficiency of health systems of autonomous communities in Spain," Public Health, vol. 138, pp. 33-40, Sep. 2016.

[46] A. Charnes, W. . Cooper, and E. Rhodes, "Measuring the efficiency of decision making units," European Journal of Operational Research, vol. 2, no. 6, pp. 429-444, Nov. 1978.

[47] R. D. Banker, A. Charnes, and W. W. Cooper, "Some Models for Estimating Technical and Scale Inefficiencies in Data Envelopment Analysis," Management Science, vol. 30, no. 9, pp. 1078-1092, Sep. 1984.

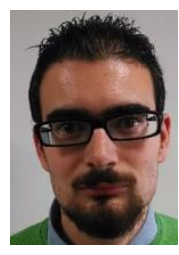

Víctor Corcoba Magaña is a Computer Science Engineer with a $\mathrm{PhD}$ from the University Carlos III, with an interest in efficient driving, embedded systems, and artificial intelligence. He is an Assistant Professor at the University of Oviedo.

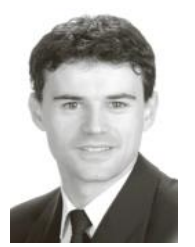

Xabiel G. Pañeda is a Computer Science Engineer with a $\mathrm{PhD}$ from the University of Oviedo, with an interest in mobile systems, content distribution networks and efficient driving. He is an Associate Professor at the University of Oviedo.

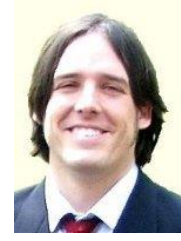

Alejandro G. Tuero (MS in Electrical Engineering, Electronics and Industrial Control) is a researcher specialized in machines and mechanisms with extensive knowledge of the automotive industry. He has been part of this research group since January 2015. Previously he had been working for companies such as Nestlé or Renault.

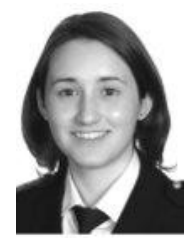

Laura Pozueco is a Telecommunications Engineer from the University of Oviedo with a PhD from the Spanish University for Distance Education (UNED). Her current research interests are in the area of telecommunication networks and services, and efficient driving.

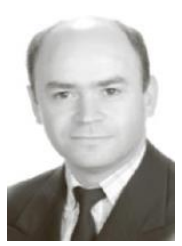

Roberto García is a Telecommunications Engineer from The Technical University of Madrid with a PhD from the University of Oviedo, with an interest in telecommunication networks and services, efficient driving, and intelligent transportation systems. $\mathrm{He}$ is an Associate Professor at the University of Oviedo.

David Melendi is a Computer Science Engineer with a PhD from the University of Oviedo, with an interest in multimedia systems, efficient driving and intelligent transportation systems. He is an Associate Professor at the University of Oviedo.

Abel Rionda has an MSc in Computer Engineering from the University of Oviedo and a $\mathrm{PhD}$ from UNED. He is CEO of ADN Mobile Solutions and his research interests are efficient driving and embedded systems. 